\title{
Optimal designs for estimating the coefficients of the lower frequencies in trigonometric regression models
}

\author{
Holger Dette \\ Ruhr-Universität Bochum \\ Fakultät für Mathematik \\ 44780 Bochum, Germany \\ e-mail: holger.dette@rub.de
}

FAX: +49 2343214559
Viatcheslav B. Melas

St. Petersburg State University

Department of Mathematics

St. Petersburg

Russia

email: v.melas@pobox.spbu.ru

\author{
P. Schpilev \\ St. Petersburg State University \\ Department of Mathematics \\ St. Petersburg \\ Russia \\ email: pitsh@font.ru
}

May 6, 2005

\begin{abstract}
In the common Fourier regression model we determine the optimal designs for estimating the coefficients corresponding to the lower frequencies. An analytical solution is provided which is found by an alternative characterization of $c$-optimal designs. Several examples are provided and the performance of the $D$-optimal design with respect to the estimation of the lower order coefficients is investigated. The results give a complete answer to an open question which was recently raised by Dette and Melas (2003).
\end{abstract}

AMS Subject classification: 62K05

Keywords and Phrases: trigonometric regression model, $c$-optimal design, Chebyshev approximation, two dimensional shape analysis

\section{Introduction}

Fourier regression models are widely used to describe periodic phenomena in applications. Typical subject areas include engineering [see e.g. McCool (1979)], medicine [see e.g. Kitsos, Titterington 
and Torsney (1988)], agronomy [see e.g. Weber and Liebig (1981)] or more generally biology [see the recent collection of research papers edited by Lestrel (1997)]. Recent applications of trigonometric regression models appear also in two dimensional shape analysis, where the coefficients of lower order are of particular importance, because they have a specific meaning in the biological context [see e.g. Young and Ehrlich (1977) and Currie et al. (2000) among many others]. It is well known that the application of an appropriate design can improve the performance of the statistical analysis in a regression model substantially and several authors have considered the problem of determining optimal designs for least squares estimation of the parameters in Fourier regression models [see e.g. Karlin and Studden (1966), page 347, Hill (1978), Lau and Studden (1985) or $\mathrm{Wu}$ (2002)]. Most authors concentrate on the estimation of the full vector of parameters and use Kiefer's $\Phi_{p}$-optimality criteria to find efficient designs for this purpose [see e.g. Pukelsheim (1993), p. 241]. Recently Dette and Melas (2003) determined optimal designs for estimating the individual coefficients corresponding to the higher frequencies in trigonometric regression models but the design problem for the estimation of the coefficients corresponding to the lower frequencies was left open. However, in many biological applications, such as two dimensional shape analysis, the coefficients corresponding to lower frequencies are usually more important, because of their concrete biological interpretation [see e.g. Young and Ehrlich (1977)]. It is the purpose of the present paper to derive an explicit solution of this problem.

The model is introduced in Section 2, where we also state some basic facts about optimal design theory. In particular a reformulation of the equivalence theorem for c-optimal designs [see e.g. Pukelsheim (1993)] is presented, which turns out to be a particularly useful tool for the solution of the optimal design problem in the present context. Our main results are stated in Section 3, where we present an explicit solution of the optimal design problem for estimating the individual coefficients corresponding to the lower frequencies in a Fourier regression model. In contrast to the designs for estimating the coefficients corresponding to the higher frequencies the optimal designs for estimating the lower order coefficients are not necessarily uniform designs. In Section 4 we present several examples illustrating our approach, while some of the technical details are deferred to an Appendix.

The optimal designs for estimating the individual coefficients advise the experimenter to take observations at a number of different locations which is smaller than the number of parameters in the model. For this reason the optimal designs derived in this paper can not be directly recommended for applications. However, we recommend to use these designs as benchmarks in evaluating the performance of commonly applied designs. Our results therefore provide an important tool for identifying efficient designs for the statistical analysis in trigonometric regression models. We illustrate this by analyzing the optimal designs in the sense of Kiefer (1974) with respect to its performance in the estimation of the individual coefficients corresponding to the lower frequencies in the trigonometric regression model. In particular it is shown that for large degree trigonometric models [as they are used in series estimation, see Eubank (1999)] these designs are rather efficient, but the loss of efficiency in lower order trigonometric regression models [as they are used in two dimensional shape analysis in biology - see Young and Ehrlich (1977) and Currie et al. (2000)] may be substantial. 


\section{Optimal designs for estimating individual coefficients}

The Fourier regression model is usually represented in the form

$$
y=\beta_{0}+\sum_{j=1}^{m} \beta_{2 j-1} \sin (j t)+\sum_{j=1}^{m} \beta_{2 j} \cos (j t)+\varepsilon, \quad x \in[-\pi, \pi],
$$

where $\varepsilon$ denotes a random variable with zero mean and positive variance, say $\sigma^{2}>0$, and different observations are assumed to be independent. We define $\beta=\left(\beta_{0}, \beta_{1}, \ldots, \beta_{2 m}\right)^{T}$ as the vector of parameters and

$$
f(t)=(1, \sin t, \cos t, \ldots, \sin (m t), \cos (m t))^{T}=\left(f_{0}(t), \ldots, f_{2 m}(t)\right)^{T}
$$

as the vector of regression functions in the model (2.1). Following Kiefer (1974) we call any probability measure $\xi$ on the design space $[-\pi, \pi]$ with finite support an (approximate) design. The support points of the design $\xi$ give the locations, where observations are taken, while the weights give the corresponding proportions of the total number of observations to be taken at these points. For uncorrelated observations [obtained from an approximate design by some rounding procedure - see e.g. Pukelsheim and Rieder (1992)] the covariance matrix of the least squares estimator for the parameter $\beta$ is approximately given by

$$
\frac{\sigma^{2}}{n} M^{-1}(\xi)
$$

where $n$ denotes the sample size and the matrix

$$
M(\xi)=\int_{-\pi}^{\pi} f(t) f^{T}(t) d \xi(t) \in \mathbb{R}^{2 m+1 \times 2 m+1}
$$

is called information matrix in the design literature. An optimal design minimizes (or maximizes) an appropriate convex (or concave) function of the information matrix and there are numerous criteria proposed in the literature, which can be used for the discrimination between competing designs [see e.g. Silvey (1980) or Pukelsheim (1993)].

In this paper we are interested in optimal designs for the estimation of the individual coefficients $\beta_{k}$ corresponding to the lower frequencies in the trigonometric regression model (2.1). To be precise let $e_{k} \in \mathbb{R}^{2 m+1}$ denote the $(k+1)$ th unit vector $(k=0, \ldots, 2 m)$ and $A^{-}$be a generalized inverse of the matrix $A \in \mathbb{R}^{2 m+1 \times 2 m+1}$; then a design $\xi$ is called $e_{k}$-optimal or optimal for estimating the coefficient $\beta_{k}$, if $\beta_{k}$ is estimable by the design $\xi$ [i.e. $e_{k} \in \operatorname{Range}(M(\xi))$ ] and $\xi$ minimizes the function

$$
\Phi_{k}(\eta)=e_{k}^{T} M^{-}(\eta) e_{k}
$$

in the set of all designs $\eta$ such that the parameter $\beta_{k}$ is estimable by the design $\eta$. $e_{k}$-optimal designs have been discussed by several authors, mainly for the case of polynomial regression on the interval $[-1,1]$ [see e.g. Studden (1968), Spruill (1990)]. Dette and Melas (2003) solved the $e_{k}$-optimal design problem for the case $k=2 \ell, k=2 \ell-1$, where $\ell>m / 3$, and for the sake of completeness we restate this result here.

Theorem 2.1. [Dette, Melas (2003)] Consider the trigonometric regression model (2.1) on the design space $[-\pi, \pi]$. 
(a) For any $\ell$ such that $m / 3<\ell \leq m$ and any $\delta \in\left[0, \frac{1}{2 \ell}\right]$ the design

$$
\xi_{2 \ell}^{*}=\left(\begin{array}{ccccc}
-\pi & -\pi+\frac{\pi}{\ell} & \ldots & -\pi+\frac{2 \ell-1}{\ell} \pi & \pi \\
\frac{1}{2 \ell}-\delta & \frac{1}{2 \ell} & \ldots & \frac{1}{2 \ell} & \delta
\end{array}\right)
$$

is optimal for estimating the parameter $\beta_{2 \ell}$. Moreover, in this case $\Phi_{2 \ell}\left(\xi_{2 \ell}^{*}\right)=1$.

(b) For any $\ell$ such that $m / 3<\ell \leq m$ the design $\xi_{2 \ell}^{*}$ defined by (2.5) is optimal for the estimation of the intercept $\beta_{0}$.

(c) For any $\ell$ such that $m / 3<\ell \leq m$ the design

$$
\xi_{2 \ell-1}^{*}=\left(\begin{array}{ccccc}
-\pi+\frac{\pi}{2 \ell} & -\pi+\frac{3 \pi}{2 \ell} & \ldots & -\pi+\frac{2 \ell-3}{2 \ell} \pi & \frac{-\pi}{2 \ell}+\pi \\
\frac{1}{2 \ell} & \frac{1}{2 \ell} & \ldots & \frac{1}{2 \ell} & \frac{1}{2 \ell}
\end{array}\right)
$$

is optimal for estimating the coefficient $\beta_{2 \ell-1}$. Moreover, in this case $\Phi_{2 \ell-1}\left(\xi_{2 \ell-1}^{*}\right)=1$.

The construction of efficient designs for estimating the lower frequencies in the trigonometric regression model appears to be substantially more difficult. In the following section we will provide an explicit solution of this problem. The main tool in our approach is a reformulation of the equivalence theorem for $c$-optimal designs as it is presented in Dette, Melas and Pepelyshev (2004) in the case of polynomial models. Because this result does not depend on a particular regression model, it is presented here for a general linear regression model of the form

$$
E[y \mid t]=\beta^{T} f(t), t \in[a, b]
$$

where the $\beta^{T}=\left(\beta_{0}, \ldots, \beta_{d}\right), f(t)=\left(f_{0}(t), \ldots f_{d}(t)\right)^{T}$. The case of the Fourier regression model (2.1) is obtained for $d=2 m$ and the choice (2.2).

Lemma 2.2. Let $\bar{f}_{k}(t)=\left(f_{0}(t), f_{1}(t), \ldots, f_{k-1}(t), f_{k+1}(t), \ldots, f_{d}(t)\right)^{T}$ denote the vector obtained from $f(t)=\left(f_{0}(t), f_{1}(t), \ldots, f_{d}(t)\right)^{T}$ by omitting the function $f_{k}(t)$. A design $\xi_{k}^{*}$ is $e_{k}$-optimal on the interval $[a, b]$ if and only if there exists a positive number $h_{k}$ and a vector $q^{*} \in \mathbb{R}^{d}$ such that the function $\varphi_{k}(t)=f_{k}(t)-\bar{f}_{k}^{T}(t) q^{*}$ satisfies the following conditions

(1) $h_{k} \varphi_{k}^{2}(t) \leq 1 \quad \forall t \in[a, b]$

(2) $\operatorname{supp}\left(\xi_{k}^{*}\right) \subset\left\{t \in[a, b] \mid h_{k} \varphi_{k}^{2}(t)=1\right\}$

(3) $\int_{a}^{b} \varphi_{k}(t) \bar{f}_{k}(t) d \xi_{k}^{*}(t)=0 \in \mathbb{R}^{d}$.

Moreover, in this case $h_{k}=\Phi_{k}\left(\xi_{k}^{*}\right)$. 


\section{Optimal designs for estimating lower frequencies}

Recall from Section 2 that Theorem 2.1 provides a solution of the $e_{2 \ell-1^{-}}$and $e_{2 \ell^{-} \text {optimal design }}$ problem whenever $m / 3<\ell \leq m$. It is also notable that the optimal designs depend rather weakly on the degree of the Fourier regression (namely only by the lastnamed inequality). For example the $e_{9^{-}}$and $e_{10^{-}}$optimal design (i.e. the optimal designs for estimating the coefficients $\beta_{9}$ or $\beta_{10}$ in the trigonometric regression model (2.1)) are the same for $m=5,6, \ldots, 14$ and do not depend on $m$. Both designs have essentially 10 support points. However, if the degree $m$ exceeds 14 the optimal designs can not be determined by Theorem 2.1 and the dependency on the degree of the Fourier regression is more severe. In particular the number of support points is substantially larger (at least 16) and the weights of the optimal designs are not necessarily equal any more. The optimal design for this case is presicely described by the main result of the present section.

Theorem 3.1 Consider the trigonometric regression model (2.1), where $m \geq 3$ and assume that $\ell \leq m / 3$.

(a) If $p=\left\lfloor\frac{m+3 \ell}{2 \ell}\right\rfloor$ is odd, then the design

$$
\xi_{2 \ell}^{*}=\left(\begin{array}{cccccc}
-t_{n} & \ldots & -t_{1} & t_{1} & \ldots & t_{n} \\
w_{n} & \ldots & w_{1} & w_{1} & \ldots & w_{n}
\end{array}\right)
$$

with $n=\ell(p-1)$,

$$
\begin{aligned}
t_{i} & =\left(2 i-1+2\left\lfloor\frac{i-1}{p-1}+\frac{1}{2}\right\rfloor\right) \frac{\pi}{2 p \ell} \quad i=1, \ldots, n \\
w_{i} & =\frac{\left|\cos t_{i}\right|}{2 \sum_{j=1}^{n}\left|\cos t_{j}\right|} \quad i=1, \ldots, n
\end{aligned}
$$

is optimal for estimating the coefficient $\beta_{2 \ell}$ in the trigonometric regression model (2.1). Moreover, the value of the optimality criterion is given by

$$
\Phi_{2 \ell}\left(\xi_{2 \ell}^{*}\right)=\left(\frac{2}{\ell p} \sum_{i=1}^{n}\left|\cos t_{i}\right|\right)^{2}=\left(\frac{2}{p} \cot \left(\frac{\pi}{2 p}\right)\right)^{2}
$$

(b) If $p=\left\lfloor\frac{m+3 \ell}{2 \ell}\right\rfloor$ is even, then any design of the form

$$
\xi_{2 \ell}^{*}=\left(\begin{array}{ccccccccc}
-\pi & -t_{n} & \ldots & -t_{2} & 0 & t_{2} & \ldots & t_{n} & \pi \\
\mu & w_{n} & \ldots & w_{2} & w_{1} & w_{2} & \ldots & w_{n} & w_{1}-\mu
\end{array}\right)
$$

with $n=\ell(p-1)$,

$$
\begin{aligned}
t_{i} & =\left(2(i-1)+2\left\lfloor\frac{i-1}{p-1}+\frac{1}{2}\right\rfloor\right) \frac{\pi}{2 p \ell} \quad i=1, \ldots, n \\
w_{i} & =\frac{\left|\cos t_{i}\right|}{2 \sum_{j=1}^{n}\left|\cos t_{j}\right|} \quad i=1, \ldots, n \\
\mu & \in\left[0, w_{1}\right]
\end{aligned}
$$


is optimal for estimating the coefficient $\beta_{2 \ell}$ in the trigonometric regression model (2.1). Moreover, the value of the optimality criterion is given by (3.3).

(c) Define $p=\left\lfloor\frac{m+3 \ell}{2 \ell}\right\rfloor, n=\ell(p-1)$, then the design

$$
\xi_{2 \ell-1}^{*}=\left(\begin{array}{cccccc}
-t_{n} & \ldots & -t_{1} & t_{1} & \ldots & t_{n} \\
w_{n} & \ldots & w_{1} & w_{1} & \ldots & w_{n}
\end{array}\right)
$$

with

$$
\begin{aligned}
t_{i} & =\left(i+\left\lfloor\frac{i-1}{p-1}\right\rfloor\right) \frac{\pi}{p \ell} \quad i=1, \ldots, n \\
w_{i} & =\frac{\left|\sin t_{i}\right|}{2 \sum_{j=1}^{n}\left|\sin t_{j}\right|} \quad i=1, \ldots, n
\end{aligned}
$$

is optimal for estimating the coefficient $\beta_{2 \ell-1}$ in the trigonometric regression model (2.1). Moreover, the value of the optimality criterion for this design is given by

$$
\Phi\left(\xi_{2 \ell-1}^{*}\right)=\left(\frac{2}{p \ell} \sum_{i=1}^{n}\left|\sin t_{i}\right|\right)^{2}=\left(\frac{2}{p} \cot \frac{\pi}{2 p}\right)^{2} .
$$

Proof. All cases are proved similarly and for this reason we restrict ourselves to the case (a) where $p=\left\lfloor\frac{m+3 \ell}{2 \ell}\right\rfloor$ is assumed to be odd. In other words we are interested in the optimal design for estimating the coefficient $\beta_{2 \ell}$ of the function $\cos (\ell t)$ where $\ell \leq \frac{m}{3}$. The proof of Theorem 3.1 is based on an explicit construction of the function $\varphi_{\ell}$ in Lemma 2.2. For this note that $p \geq 3$ (because $\ell \leq m / 3$ ) and consider the trigonometric polynomial

$$
L(t, a)=\sum_{j=1}^{p-1} a_{j} \cos ((2 j-1) \ell t)
$$

where $\left(a_{1}, \ldots, a_{p-1}\right)^{T} \in \mathbb{R}^{p-1}$ is a given vector. We determine this vector such that the function $L$ satisfies

$$
\begin{array}{rl}
L\left(t_{i}, a\right)=\frac{\cos \left(\ell t_{i}\right)}{\left|\cos \ell t_{i}\right|} & i=1, \ldots, \frac{p-1}{2} \\
L^{\prime}\left(t_{i}, a\right)=0 & i=1, \ldots, \frac{p-1}{2}
\end{array}
$$

where the points $t_{1}, \ldots, t_{\frac{p-1}{2}}$ are defined by (3.1). It is easy to see that the system of equations in (3.10) is equivalent to the system

$$
B a=e,
$$


where the vector $e \in \mathbb{R}^{p-1}$ is given by

$$
e=\left(\frac{\cos \left(\ell t_{1}\right)}{\left|\cos \left(\ell t_{1}\right)\right|}, \ldots, \frac{\cos \left(\ell t_{\frac{p-1}{2}}\right)}{\left|\cos \left(\ell t_{\frac{p-1}{2}}\right)\right|}, 0, \ldots, 0\right)^{T}
$$

and the $(p-1) \times(p-1)$ matrix $B$ is defined as

$$
B=\left(\begin{array}{cccc}
\cos \left(\ell t_{1}\right) & \cos \left(3 \ell t_{1}\right) & \cdots & \cos \left((2 p-3) \ell t_{1}\right) \\
\vdots & \vdots & \vdots & \vdots \\
\cos \left(\ell t_{\frac{p-1}{2}}\right) & \cos \left(3 \ell t_{\frac{p-1}{2}}\right) & \cdots & \cos \left((2 p-3) \ell t_{\frac{p-1}{2}}\right) \\
-\ell \sin \left(\ell t_{1}\right) & -3 \ell \sin \left(3 \ell t_{1}\right) & \cdots & -(2 p-3) \ell \sin \left((2 p-3) \ell t_{1}\right) \\
\vdots & \vdots & \vdots & \vdots \\
-\ell \sin \left(\ell t_{\frac{p-1}{2}}\right) & -3 \ell \sin \left(3 \ell t_{\frac{p-1}{2}}\right) & \cdots & -(2 p-3) \ell \sin \left((2 p-3) \ell t_{\frac{p-1}{2}}\right)
\end{array}\right) .
$$

It follows from Lemma A.1 in the Appendix that $\operatorname{det} B \neq 0$. Therefore the system of equation in (3.11) or equivalently in (3.10) has a unique solution, say $\hat{a}=\left(\hat{a}_{1}, \ldots, \hat{a}_{p-1}\right)^{T}$. In the following we investigate the properties of the function $L(t, \hat{a})$ corresponding to this solution. In order to simplify the notation we assume $\ell=1$ throughout the remaining part of the proof. The general case can be proved exactly in the same way and the (minor) differences will be briefly mentioned at the corresponding places.

We will show in Lemma A.2 in the Appendix that the function $L(t, \hat{a})$ satisfies

$$
|L(t, \hat{a})| \leq 1 \quad \forall t \in[-\pi, \pi]
$$

From the orthonormality conditions

$$
\frac{2}{\pi} \int_{0}^{\pi} \cos (i t) \cos (j t) d t=\left\{\begin{array}{l}
1 \text { if } i=j \neq 0 \\
0 \text { if } i \neq j
\end{array}\right.
$$

we obtain that the coefficient of $\cos t$ in the function $L(t, \hat{a})$ has the representation

$$
\hat{a}_{1}=\frac{2}{\pi} \int_{0}^{\pi} L(t, \hat{a}) \cos t d t
$$

In order to calculate this expression explicitly we will use the fact that the points

$$
x_{i}=\cos u_{i}=\cos \left(\frac{2 i-1}{2 p} \pi\right), \quad i=1, \ldots, p
$$

and weights $\alpha_{i}=\frac{1}{p}(i=1, \ldots, p)$ define a quadrature formula of degree $2 p-1$ on the interval with respect to the arcsine distribution, that is

$$
\frac{1}{\pi} \int_{-1}^{1} P_{k}(x) \frac{d x}{\sqrt{1-x^{2}}}=\frac{1}{\pi} \int_{0}^{\pi} P_{k}(\cos t) d t=\sum_{i=1}^{p} P_{k}\left(x_{i}\right) \alpha_{i}=\sum_{i=1}^{p} \alpha_{i} P_{k}\left(\cos u_{i}\right)
$$


for all polynomials $P_{k}$ of degree $k \leq 2 p-1$ [see Stroud and Secrest (1966)]. Since

$$
2 p-1=2\left\lfloor\frac{m+3}{2}\right\rfloor-1=2\left\lfloor\frac{m+1}{2}\right\rfloor+1 \geq m+1
$$

and the function $L(t, \hat{\alpha}) \cos t$ can be represented in the form $P_{m+1}(\cos t)$, where the degree of $P_{m+1}$ is at most $m+1$, we can use the quadrature formula (3.17) to evaluate the expression in (3.15). For this we note that by the definition of the points $t_{i}$ and $u_{i}$ in (3.1) and (3.16), respectively, we have

$$
\begin{array}{ll}
u_{i}=\frac{2 i-1}{2 p} \pi=t_{i} & \text { if } i=1, \ldots, \frac{p-1}{2}, \\
u_{i}=\frac{\pi}{2} & \text { if } i=\frac{p+1}{2}, \\
u_{i}=t_{i-1} & \text { if } i=\frac{p+3}{2}, \ldots, p
\end{array}
$$

which yields, by the definition of the function $L(t, \hat{a})$,

$$
L\left(u_{i}, \hat{a}\right)=\left\{\begin{array}{cl}
\frac{\cos u_{i}}{\left|\cos u_{i}\right|} & \text { if } i \neq \frac{p+1}{2} \\
0 & \text { if } i=\frac{p+1}{2} .
\end{array}\right.
$$

This gives for the coefficient $\hat{a}_{1}$ in $(3.15)$

$$
\begin{aligned}
\hat{a}_{1} & =\frac{2}{\pi} \int_{0}^{\pi} L(t, \hat{a}) \cos t d t=\frac{2}{p} \sum_{i=1}^{p} L\left(u_{i}, \hat{a}\right) \cos u_{i} \\
& =\frac{2}{p} \sum_{i=1}^{p}\left|\cos u_{i}\right|=\frac{2}{p} \sum_{i=1}^{p-1}\left|\cos t_{i}\right| .
\end{aligned}
$$

Consider now the function

$$
\varphi(t)=\frac{L(t, \hat{a})}{\hat{a}_{1}}
$$

and note that the coefficient of $\cos t$ is 1 . Therefore the function $\varphi$ can be represented as

$$
\varphi(t)=\cos t-\tilde{a}^{T} \bar{f}_{2}(t)
$$

with a suitable vector $\tilde{a}$ and

$$
\bar{f}_{2}(t)=(1, \sin t, \sin 2 t, \cos 2 t, \ldots, \sin (m t) \cos (m t))^{T} .
$$

We will now show that the conditions of Lemma 2.2 are satisfied for the function $\varphi$. For this we define $h=\hat{a}_{1}^{2}$ and obtain from (3.14) and (3.20)

$$
h \varphi^{2}(t) \leq 1
$$

for all $t \in[-\pi, \pi]$. Moreover, it follows from the construction of the function $L$ that there is equality in (3.23) if $t=t_{i}$ or $t=-t_{i}$ for $i=1, \ldots, n$. Therefore the function $\varphi$ satisfies the 
conditions (1) and (2) of Lemma 2.2. In order to prove the remaining condition (3) of this Lemma we note $\varphi\left(t_{i}\right)=\varphi\left(-t_{i}\right), i=1, \ldots, n$, which implies

$$
\int_{-\pi}^{\pi} \varphi(t) \sin (j t) d \xi_{2}^{*}(t)=\sum_{i=1}^{n}\left\{\varphi\left(t_{i}\right) \sin \left(j t_{i}\right)+\varphi\left(-t_{i}\right) \sin \left(-j t_{i}\right)\right\} w_{i}=0
$$

for $j=1, \ldots, m$. Moreover, from (3.17) we derive the well known relation

$$
\sum_{i=1}^{p} \cos \left(k \frac{2 i-1}{2 p} \pi\right)=0, \quad k=1,2, \ldots, 2 p-1
$$

which imply [note that $n=p-1$ and observe the relation (3.10), (3.18) and (3.19)]

$$
\begin{aligned}
\int_{-\pi}^{\pi} \varphi(t) \cos (j t) d \xi_{2}^{*}(t) & =2 \sum_{i=1}^{n} \varphi\left(t_{i}\right) \cos \left(j t_{i}\right) w_{i} \\
& =\sum_{i=1}^{n} \frac{L\left(t_{i}, \hat{a}\right)}{\hat{a}_{1}} \cos \left(j t_{i}\right) \frac{\left|\cos t_{i}\right|}{\sum_{j=1}^{n}\left|\cos t_{j}\right|} \\
& =\frac{2}{p \hat{a}_{1}^{2}} \sum_{i=1}^{p} \cos \left(\frac{2 i-1}{2 p} \pi\right) \cos \left(j \pi \frac{2 i-1}{2 p}\right) \\
& =\frac{1}{p \hat{a}_{1}^{2}} \sum_{i=1}^{p}\left\{\cos \left((j-1) \frac{2 i-1}{2 p} \pi\right)+\cos \left((j+1) \frac{2 i-1}{2 p} \pi\right)\right\}=0
\end{aligned}
$$

for $j=2,3, \ldots, 2 p-2$. Since $m \leq 2 p-2$ it therefore follows from these relations that

$$
\int \varphi(t) \bar{f}_{2}(t) d \xi_{2}^{*}(t)=0 \in \mathbb{R}^{2 m}
$$

which proves the remaining assumption (3) of Lemma 2.2. By this result it now follows that the design $\xi_{2}^{*}$ is optimal for estimating the coefficient $\beta_{2}$ in the trigonometric regression model (2.5). For a proof of the identity in (3.3) we note that it follows from formula 420 in Jolley (1961) that

$$
\begin{aligned}
\sum_{i=1}^{n}\left|\cos t_{i}\right| & =2 \sum_{i=1}^{\left\lfloor\frac{p-1}{2}\right\rfloor} \cos t_{i}=2 \sum_{i=1}^{\left\lfloor\frac{p-1}{2}\right\rfloor} \cos \left(\frac{2 i-1}{2 p} \pi\right) \\
& =\frac{\sin \left(\frac{(p-1) \pi}{2 p}\right)}{\sin \left(\frac{\pi}{2 p}\right)}=\cot \left(\frac{\pi}{2 p}\right) .
\end{aligned}
$$

This proves part (a) of Theorem 3.1 in the case $\ell=1$. The general case $\ell \geq 2$ follows by exactly the same arguments with an additional amount of notation in this proof. Finally, the cases (b) and (c) are shown similarly and the proof is therefore omitted. 


\section{Examples}

In this section we briefly illustrate the results of Section 3 by calculating explicitly the optimal designs for estimating the coefficients corresponding to the lower frequencies in a Fourier regression model (2.1) of degree $m=3,4,5,6$.

Example 4.1. Consider a Fourier regression model of degree $m=3$ or $m=4$. The optimal designs for estimating the coefficients $\beta_{0}$ and $\beta_{i}(i \geq 3)$ can be directly obtained from Theorem 2.1 and are not given here for the sake of brevity. For the construction of the two remaining optimal designs we begin with the optimal design for estimating the coefficient $\beta_{1}$ (that is $\ell=1$ ) and note that in this case

$$
p=\left\lfloor\frac{m+3 \ell}{2 \ell}\right\rfloor=\left\lfloor\frac{m+3}{2}\right\rfloor=3
$$

for $m=3,4$. By Theorem 3.1(c) the points $t_{1}, t_{2}$ are given by $t_{1}=\pi / 3, t_{2}=2 \pi / 3$ which gives $\sin t_{1}=\sin t_{2}=\sqrt{3} / 2$. Therefore the optimal design for estimating the coefficient $\beta_{1}$ in the trigonometric regression model (2.5) with degree $m=3,4$ is given by

$$
\xi_{1}^{*}=\left(\begin{array}{cccc}
-\frac{2 \pi}{3} & -\frac{\pi}{3} & \frac{\pi}{3} & \frac{2 \pi}{3} \\
\frac{1}{4} & \frac{1}{4} & \frac{1}{4} & \frac{1}{4}
\end{array}\right)
$$

and the value of the optimality criterion is $\Phi_{1}\left(\xi_{1}^{*}\right)=\frac{4}{3}$. Finally, the function $\varphi$ required for the application of Lemma 2.2 is given by

$$
\varphi(t)=\sin t+\frac{1}{6} \sin (3 t)
$$

In order to determine the optimal design for estimating the coefficient $\beta_{2}$ we use the first part of Theorem 3.2 and obtain $t_{1}=\pi / 6, t_{2}=\pi / 6$. Observing that $\cos t_{1}=-\cos t_{2}=\sqrt{3} / 2$ it follows that

$$
\xi_{2}^{*}=\left(\begin{array}{cccc}
-\frac{5}{6} \pi & -\frac{\pi}{6} & \frac{\pi}{6} & \frac{5}{6} \pi \\
\frac{1}{4} & \frac{1}{4} & \frac{1}{4} & \frac{1}{4}
\end{array}\right)
$$

is an optimal design for estimating the coefficient $\beta_{2}$ in the trigonometric regression of degree $m=3,4$. The value of the optimality criterion is again $\Phi_{2}\left(\xi_{2}^{*}\right)=\frac{4}{3}$ and the function required for the application of in Lemma 2.2 is given by

$$
\varphi(t)=\cos t-\frac{1}{6} \cos 3 t
$$

Example 4.2. Consider the Fourier regression model (2.5) of degree $m=5$ and 6 . If $m=5$ only the designs for estimating the individual coefficients $\beta_{1}$ and $\beta_{2}$ can not be obtained from Theorem 2.1. The optimal design for estimating the coefficient $\beta_{1}$ in the trigonometric regression model (2.5) with $m=5$ is given by

$$
\xi_{1}^{*}=\left(\begin{array}{cccccc}
-\frac{3 \pi}{4} & -\frac{\pi}{2} & -\frac{\pi}{4} & \frac{\pi}{4} & \frac{\pi}{2} & \frac{3 \pi}{4} \\
\frac{1}{4+2 \sqrt{2}} & \frac{\sqrt{2}}{4+2 \sqrt{2}} & \frac{1}{4+2 \sqrt{2}} & \frac{1}{4+2 \sqrt{2}} & \frac{\sqrt{2}}{4+2 \sqrt{2}} & \frac{1}{4+2 \sqrt{2}}
\end{array}\right)
$$




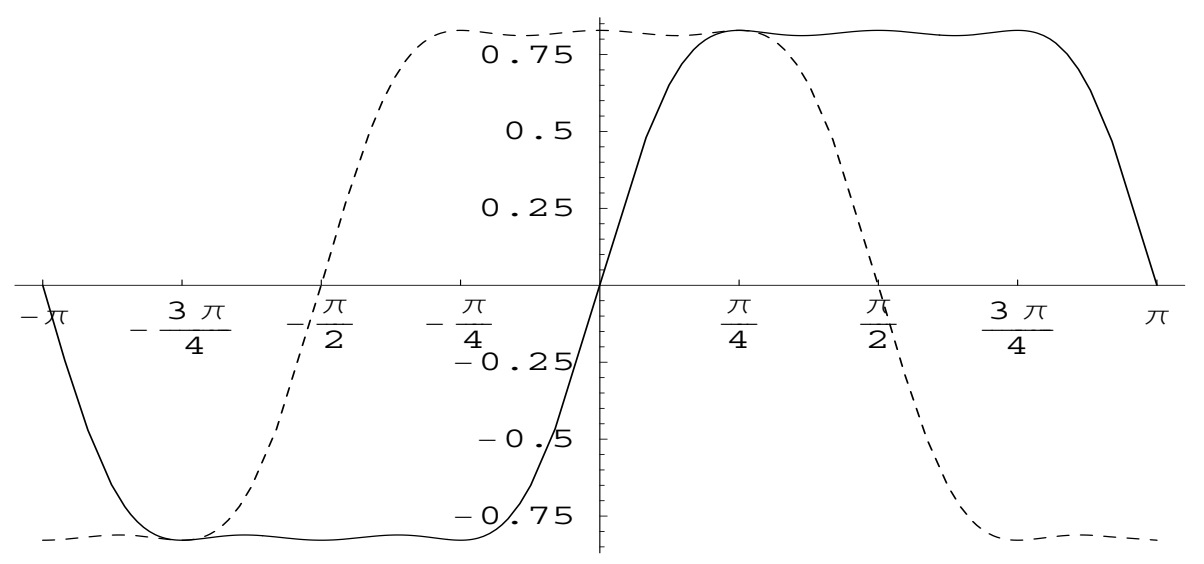

Figure 4.1: The functions defined in Lemma 2.2 corresponding to the estimation of the coefficients $\beta_{1}$ and $\beta_{2}$ in a Fourier regression model of degree $m=5$, respectively. The functions are defined explicitly by (4.2) (solid line) and (4.4) (dotted line).

and the function required for the application of Lemma 2.2 is

$$
\varphi(t)=\sin t+\frac{8-5 \sqrt{2}}{4} \sin (3 t)+\frac{3 \sqrt{2}-4}{4} \sin (5 t) .
$$

The value of the optimality criterion is obtained as $\Phi\left(\xi_{1}^{*}\right)=\frac{3+2 \sqrt{2}}{4}$. The optimal design for estimating the coefficient $\beta_{2}$ is given by

$$
\xi_{2}^{*}=\left(\begin{array}{ccccccc}
-\pi & -\frac{3 \pi}{4} & -\frac{\pi}{4} & 0 & \frac{\pi}{4} & \frac{3 \pi}{4} & \pi \\
\mu & \frac{\sqrt{2}}{4+4 \sqrt{2}} & \frac{\sqrt{2}}{4+4 \sqrt{2}} & \frac{2}{4+4 \sqrt{2}} & \frac{\sqrt{2}}{4+4 \sqrt{2}} & \frac{\sqrt{2}}{4+4 \sqrt{2}} & \frac{2}{4+4 \sqrt{2}}-\mu
\end{array}\right)
$$

where $\mu \in[0,2 /(4+4 \sqrt{2})]$ is arbitrary. The corresponding function in Lemma 2.2 is given by

$$
\varphi(t)=\cos t-\frac{8-5 \sqrt{2}}{4} \cos (3 t)-\frac{3 \sqrt{2}-4}{4} \cos (5 t) .
$$

The polynomials in (4.2) and (4.4) are depicted in Figure 4.1.

In the trigonometric regression model of degree $m=6$, the optimal designs for estimating the coefficients $\beta_{1}, \beta_{2}, \beta_{3}, \beta_{4}$ can not be found by an application of Theorem 2.1, and Theorem 3.1 has to be used for this purpose. The optimal designs for estimating the coefficients $\beta_{1}$ and $\beta_{2}$ are given by (4.1) and (4.3), respectively. The optimal design for estimating the coefficient $\beta_{3}$ is a uniform distribution at the 8 points

$$
\pm \frac{\pi}{6}, \pm \frac{2 \pi}{6}, \pm \frac{4 \pi}{6}, \pm \frac{5 \pi}{6},
$$

while the corresponding function in Lemma 2.2 is given by

$$
\varphi(t)=\sin (2 t)+\frac{1}{6} \sin (6 t),
$$


and the value of the optimality criterion is $\Phi\left(\xi_{3}^{*}\right)=\frac{4}{3}$. Finally, the design for estimating the coefficient $\beta_{4}$ is given by a uniform distribution at the points

$$
\pm \frac{11 \pi}{12}, \pm \frac{7 \pi}{12}, \pm \frac{5 \pi}{12}, \pm \frac{\pi}{12}
$$

the corresponding function in Lemma 2.2 is $\varphi(t)=\cos (2 t)-\frac{1}{6} \cos (6 t)$ and the value of the optimality criterion is again $\Phi\left(\xi_{4}^{*}\right)=\frac{4}{3}$.

Example 4.3. Commonly used designs for trigonometric regression models are uniform designs with at least $n \geq 2 m+1$ different points. As pointed out by Pukelsheim (1993) these designs are optimal with respect to Kiefer's $\Phi_{p}$-criteria for the least squares estimation of the full vector of parameters. It is therefore of interest to investigate the performance of these designs with respect to the estimation of the coefficients corresponding to the lower frequencies in the trigonometric regression model (2.5). For this purpose we note that the information matrix of a uniform design, say $\xi_{u}$, in this model is given by

$$
M\left(\xi_{u}\right)=\operatorname{diag}(1,2, \ldots, 2) \in \mathbb{R}^{2 m+1 \times 2 m+1},
$$

[see Pukelsheim (1993)] and therefore the efficiency of the uniform design for estimating the coefficient $\beta_{k}(k \geq 1)$ is obtained as

$$
\operatorname{eff}_{k}\left(\xi_{u}\right)=\frac{\Phi_{k}\left(\xi_{u}\right)}{\Phi_{k}\left(\xi_{k}^{*}\right)}=\left(2-\delta_{k 0}\right)\left(\frac{2}{p} \cot \left(\frac{\pi}{2 p}\right)\right)^{-2}
$$

where $k=2 \ell, 2 \ell-1, \ell \leq m / 3$ and $p=\left\lfloor\frac{m+3 \ell}{2 \ell}\right\rfloor$. For example, if $m=6$ and $k=4(\ell=2)$ or $k=3(\ell=2)$ we obtain

$$
\operatorname{eff}_{3}\left(\xi_{u}\right)=\operatorname{eff}_{4}\left(\xi_{u}\right)=\frac{3}{2}
$$

which shows that the loss of efficiency for estimating the coefficients $\beta_{3}$ and $\beta_{4}$ in a trigonometric regression model of degree $m=6$ is $50 \%$ if a uniform design is used. However, if the degree of the trigonometric regression is larger, then the uniform design has a better efficiency. In particular, if $m \rightarrow \infty$ we obtain

$$
\lim _{p \rightarrow \infty} \frac{2}{p} \cot \left(\frac{\pi}{2 p}\right)=\frac{4}{\pi}
$$

which gives for the efficiency of the uniform design

$$
\lim _{m \rightarrow \infty} \operatorname{eff}_{k}\left(\xi_{u}\right)=\frac{\pi^{2}}{8} \approx 1.235, \quad k \geq 1
$$

(note that asymptotically the limit does not depend on $k$ ). In other words if the uniform design is used in a trigonometric regression with a large degree [as it appears in series estimation, see Eubank (1999)] the loss of efficiency for estimating the coefficients corresponding to the lower frequencies is $23.5 \%$. However, if the uniform design is used in a trigonometric regression model of lower degree [as it appears in two dimensional shape analysis, see Young and Ehrlich (1977) or Currie et al. (2000)] the loss of efficiency is more substantial. 


\section{Appendix: some technical results}

Lemma A.1. The matrix $B$ defined by (3.13) is nonsingular.

Proof. Let $s \in \mathbb{N}$ and consider the matrix

$$
A=\left(\begin{array}{cccc}
\cos t_{1} & \cos \left(3 t_{1}\right) & \ldots & \cos \left((4 s-1) t_{1}\right) \\
\cos t_{2} & \cos \left(3 t_{2}\right) & \ldots & \cos \left((4 s-1) t_{2}\right) \\
\vdots & \vdots & \vdots & \vdots \\
\cos t_{2 s} & \cos \left(3 t_{2 s}\right) & \ldots & \cos \left((4 s-1) t_{2 s}\right)
\end{array}\right)=\left(u_{1}, \ldots, u_{2 s}\right) \in \mathbb{R}^{2 s \times 2 s}
$$

whee $t_{1}<t_{2}<\ldots<t_{2 s}$ are arbitrary numbers and the vector $u_{\ell}$ is defined by

$$
u_{\ell}=\left(\cos \left((2 \ell-1) t_{1}\right), \ldots, \cos \left((2 \ell-1) t_{2 s}\right)\right)^{T} \quad \ell=1, \ldots, 2 s .
$$

Let $U_{2 \ell-1}(x)=\sin (2 \ell \arccos x) / \sin (\arccos x)$ denote the Chebyshev polynomial of the second kind [see Szegö (1975)]. Observing the identity

$$
\sum_{j=1}^{\ell} \cos ((2 j-1) t)=\frac{1}{2} \frac{\sin (2 \ell t)}{\sin t}=\frac{1}{2} U_{2 \ell-1}(x)
$$

with $x=\cos t$ [see Jolley (1961), formula 420]. This implies

$$
\begin{aligned}
\operatorname{det} A & =\operatorname{det}\left(u_{1}, u_{1}+u_{2}, u_{1}+u_{2}+u_{3}, \ldots, u_{1}+\ldots+u_{2 s}\right) \\
& =\left(\frac{1}{2}\right)^{2 s} \operatorname{det}\left(U_{2 j-1}\left(x_{i}\right)\right)_{i, j=1}^{2 s}
\end{aligned}
$$

where $x_{i}=\cos t_{i}(i=1, \ldots, 2 s)$. It is well known that the leading coefficient of $U_{j}(x)$ is $2^{j}$ [see Szegö (1975)], and we obtain (using the Vandermonde determinant formula)

$$
\begin{aligned}
\operatorname{det} A & =\left(\frac{1}{2}\right)^{2 s} 2^{\sum_{j=1}^{2 s}(2 j-1)} \operatorname{det}\left(x_{i}^{2 j-1}\right)_{i, j=1}^{2 s} \\
& =2^{2 s(2 s-1)} \prod_{i=1}^{2 s} \cos t_{i} \prod_{1 \leq i<j \leq 2 s}\left(\cos ^{2} t_{j}-\cos ^{2} t_{i}\right) .
\end{aligned}
$$

Subtracting the $i$ th row of the matrix $A$ from the $(i+1)$ th row $(i=1, \ldots, s)$ it follows that the determinant of the matrix $B$ defined by (3.13) can be obtained as

$$
\begin{aligned}
\operatorname{det} B & =\lim _{t_{i+s} \rightarrow t_{i} ; i=1, \ldots, s} \frac{\operatorname{det} A}{\prod_{i=1}^{s}\left(t_{i+s}-t_{i}\right)} \\
& =-2^{2 s(2 s-1)} \prod_{i=1}^{s}\left(\cos ^{2} t_{i} \sin 2 t_{i}\right) \prod_{1 \leq i<j \leq s}\left(\cos ^{2} t_{i}-\cos ^{2} t_{j}\right)^{4} .
\end{aligned}
$$

Finally, if $s \geq \frac{p-1}{2}$ and the points $t_{1}, \ldots, t_{\frac{p-1}{2}}$ are given by (3.1) it follows that $t_{j} \in\left(0, \frac{\pi}{2}\right)(j=$ $1, \ldots, \frac{p-1}{2}$ ) which implies $\operatorname{det} B \neq 0$. 
Lemma A.2. The polynomial $L(t, \hat{a})$ defined by (3.9) and (3.11) satisfies the inequality (3.14).

Proof. Recall that $p$ is assumed as odd. Note that the system

$$
\{\sin t, \sin (3 t), \ldots, \sin ((2 p-1) t)\}
$$

is a Chebyshev system on the interval $\left(0, \frac{\pi}{2}\right)$ and consequently the function

$$
L^{\prime}(t, \hat{a})=-\sum_{j=1}^{p-1}(2 j-1) \hat{a}_{j} \sin ((2 j-1) t)
$$

has at most $p-2$ roots in the interval $\left(0, \frac{\pi}{2}\right)$ counted with their multiplicities [see Karlin and Studden (1966)]. Therefore a careful counting of the multiplicities yields that the function $L(t, \hat{a})$ has exactly one extreme point in each of the intervals $\left(t_{i}, t_{i+1}\right) ; i=1, \ldots, \frac{p-3}{2}$.

Moreover, by (3.10) we have $\hat{L}\left(t_{i}, \hat{a}\right)=1$, if $i=1, \ldots, \frac{p-1}{2}$, and in follows from the Chebyshev property of the system $\{\cos t, \cos (3 t), \ldots, \cos ((2 p-3) t)\}$ on the interval $\left(0, \frac{\pi}{2}\right)$ that there is exactly one local minimum in the intervals $\left(t_{i}, t_{i+1}\right) ; i=1, \ldots, \frac{p-3}{2}$. Now the system

$$
\{\sin t, \sin (2 t), \ldots, \sin ((2 p-3) t)\}
$$

is a Chebyshev system on the interval $(0, \pi)$ and therefore the function in (A.1) has at most $(2 p-4)$ roots in this interval (counted with their multiplicities). By the above discussion there remain $(p-2)$ roots (counted with their multiplicities) and by (3.10)

$$
L\left(t_{i}, \hat{a}\right)=-1 ; i=\frac{p+1}{2}, \ldots, p-1 .
$$

Again a careful counting of the multiplicities of the roots shows that there is exactly one local maximum in the intervals

$$
\left(t_{i}, t_{i+1}\right), i=\frac{p+1}{2}, \ldots, p-2 .
$$

Note that in the case $p<5$ there do not exist such intervals.

Now assume that there exists a point $t^{*} \in[-\pi, \pi]$ such that $\left|L\left(t^{*}, \hat{a}\right)\right|>1$. A direct calculation shows that this is not possible in the case $p=3$ and we can assume $p \geq 5$ in the remaining part of the proof. By the discussion of the previous paragraph there exists a point

$$
\bar{t}=\min \left\{\left|t^{*}\right|, \pi-\left|t^{*}\right|\right\} \in\left(0, \frac{\pi}{2}\right)
$$

such that

$$
L(\bar{t}, \hat{a})<-1
$$

and $\bar{t} \in\left(t_{i}, t_{i+1}\right)$ for some $i \in\left\{1, \ldots, \frac{p-3}{2}\right\}$. We now compare the function $L(t, \hat{a})$ with the function $\cos (2 p t)$ [in the case $\ell>1$ one has to use the function $\cos (2 p \ell t)$ ]. For this purpose we define the trigonometric polynomial

$$
Q(t)=L(t, \hat{a})-\cos (2 p t) .
$$




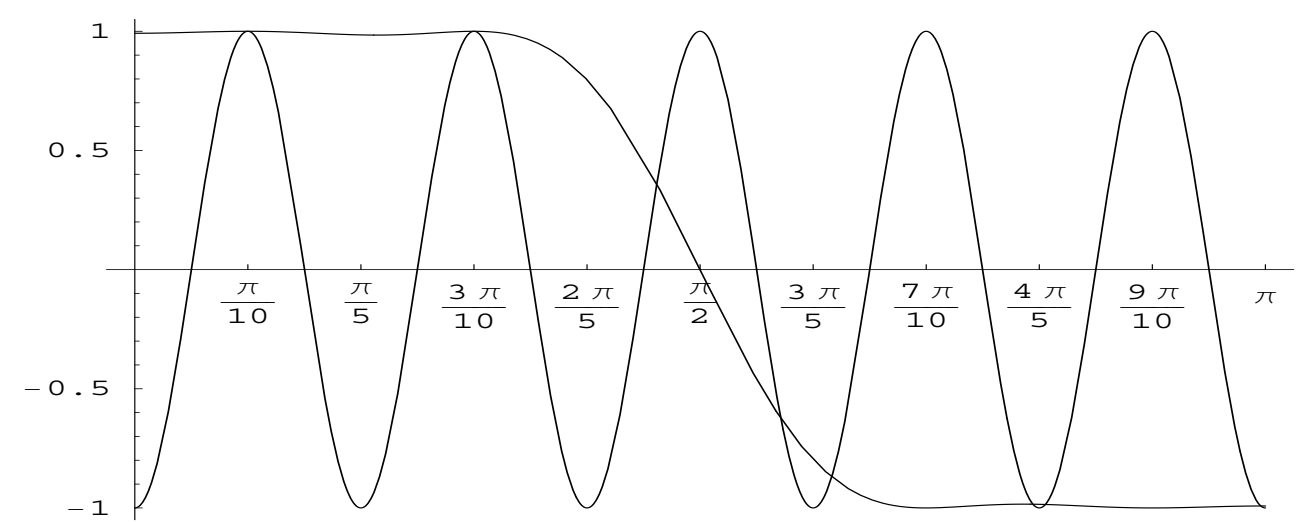

Figure 4.2: The functions $L(t, \hat{a})$ defined by (3.10) and the function $t \rightarrow \cos (2 p t)$ in the case $p=5$. The polynomial $Q(t)$ defined by (A.4) has two roots of multiplicity 2 at $t_{1}=\frac{\pi}{10}, t_{2}=\frac{3 \pi}{10}$ and both graphs intersect in each of the intervals $\left((j-1) \frac{\pi}{10}, j \frac{\pi}{10}\right) \quad j=5, \ldots, 10$.

Because the functions

$$
1, \cos t, \cos (2 t), \ldots, \cos (2 p t)
$$

form a Chebyshev system on the interval $[0, \pi]$, it follows that the function $Q(t)$ has at most $2 p$ roots in the interval $[0, \pi]$ counted with their multiplicities. From the definition of the function $L$ in (3.10) we obtain that the points $t_{1}, \ldots, t_{\frac{p-1}{2}}$ are roots of the trigonometric polynomial $Q$ and their multiplicity is at least two. Moreover, since $L(t, \hat{a})$ changes in the interval $\left((p-1) \frac{\pi}{2 p}, \frac{\pi}{2}\right)$ from +1 to 0 and $-\cos (2 p t)$ changes from -1 to 1 , the function has a further root in the interval $\left((p-1) \frac{\pi}{2 p}, \frac{\pi}{2}\right)$. By a similar argument it follows that the function $Q$ has a root in each of the $p$ intervals

$$
\left(\frac{\pi}{2},(p+1) \frac{\pi}{2 p}\right),\left((p+1) \frac{\pi}{2 p},(p+2) \frac{\pi}{2 p}\right), \ldots,\left((2 p-1) \frac{\pi}{2 p}, \pi\right)
$$

(note that $\cos (2 p t)$ attains each value in $[-1,1]$ in each of these intervals ). Consequently, there do not exist any other roots of the trigonometric polynomial $Q$. A typical picture of the function $L$ and the function $-\cos (2 p t)$ is displayed in Figure 1 for the case $p=5$.

By our assumption the point $\bar{t}$ defined in (A.2) satisfies (A.3) and is located in one of the intervals $\left(t_{i}, t_{i+1}\right)$, where $i \in\left\{1, \ldots, \frac{p-3}{2}\right\}$. But this would mean that the function $Q$ has two additional roots in the interval $\left(t_{i}, t_{i+1}\right)$ yielding to a total number of $2 p+2$ roots (counted with their multiplicities). This is a contradition to the Chebyshev property of the system in (A.5) and shows that $|L(t, \hat{a})| \leq 1$ for all $t \in[0, \pi]$. By the symmetry of the function $L$ this property also holds for the interval $[-\pi, 0]$, which completes the proof of Lemma A.2.

Acknowledgements. The work of the authors was supported by the Deutsche Forschungsgemeinschaft (SFB 475 Komplexitätsreduktion in multivariaten Datenstrukturen). The authors would also like to thank Isolde Gottschlich, who typed this paper with considerable technical expertise. 


\section{References}

A.J. Currie, S. Ganeshanandam, D.A. Noiton, D. Garrick. C.J.A. Shelbourne, N. Oragguzie (2000). Quantiative evluation of apple (Malus x domestica Borkh.) fruit shape by prinicple component analysis of Fourier descriptors. Euphytica 111, 219-227.

H. Dette, V.B. Melas (2003). Optimal designs for estimating individual coefficients in Fourier regression models. Ann. Statist. 31, 1669-1692.

H. Dette, V.B. Melas, A. Pepelysheff (2004). Optimal designs for estimating individual coefficients - a functional approach. J. Stat. Plann. Inference 118, 201-219.

R. Eubank (1999). Nonparametric regression and spline smoothing. 2nd ed. Marcel Dekker.

L.B.W. Jolley (1961). Summation of series. New York: Dover Publications, Inc.

P.D.H. Hill (1978). A note on the equivalence of $D$-optimal design measures for three rival linear models. Biometrika 65, 666-667.

S. Karlin, W.J. Studden (1966). Tchebycheff systems: with applications in analysis and statistics. Interscience, New York.

J.C. Kiefer (1974). General equivalence theory for optimum designs (approximate theory). Ann. Statist. 2, 849-879.

C.P. Kitsos, D.M. Titterington, B. Torsney (1988). An optimal design problem in rhythmometry. Biometrics 44, 657-671.

T.S. Lau, W.J. Studden (1985). Optimal designs for trigonometric and polynomial regression. Ann. Statist. 13, 383-394.

P.E. Lestrel (1997). Fourier descriptors and their applications in biology. Cambridge Univ. Press. J.I. McCool (1979). Systematic and random errors in least squares estimation for circular contours. Precision Engineering 1, 215-220.

F. Pukelsheim (1993). Optimal Design of Experiments. Wiley, New York.

F. Pukelsheim, S. Rieder (1992). Efficient rounding of approximate designs. Biometrika 79, 763770 .

S.D. Silvey (1980). Optimal design. Chapman and Hall, London.

M.C. Spruill (1990). Good designs for testing the degree of a polynomial mean. Sankhya, Ser. B $52,67-74$

A.H. Stroud, D. Secrest (1966). Gaussian quadrature formulas. Prentice and Hall, London.

W.J. Studden (1968). Optimal design on Tchebycheff points. Ann. Math. Statist. 39, 1435-1447.

G. Szegö (1959). Orthogonal polynomials. Am. Math. Soc. Colloqu. Publ. 23. Providence, RI.

W.E. Weber, H.P. Liebig (1981). Fitting response functions to observed data. Elektronische Datenverarbeitung in Medizin und Biologie 12, 88-92.

$\mathrm{H}$. $\mathrm{Wu}$ (2002). Optimal designs for first-order trigonometric regression on a partial circle. Statist. Sinica 12, 917-930.

J.C. Young, R. Ehrlich (1977). Fourier biometrics: harmonic amplitudes as multivariate shape descriptors. Syst. Zool. 26, 336-342. 